\title{
Maternal serum pentraxin 3 level in early pregnancy for prediction of gestational diabetes mellitus
}

\author{
Xiaoxian $\mathrm{Qu}^{1 *}$, Jingyi Zhuang ${ }^{1 *}$, Chuanlu $\mathrm{Xu}^{1}{ }^{1}$, Zisheng $\mathrm{Ai}^{2}$, Ling Yuan ${ }^{1}$, Yuping Tang ${ }^{1}$, Qun Shu ${ }^{1}$, Yirong Bao ${ }^{1}$, \\ Huan Han ${ }^{1}$, Hao Ying ${ }^{1}$ \\ ${ }^{1}$ Department of Obstetrics, Shanghai First Maternity and Infant Hospital, Tongji University School of Medicine, Shanghai 201204, China; \\ ${ }^{2}$ Department of Medical Statistics, Tongji University School of Medicine, Shanghai 200092, China \\ Contributions: (I) Conception and design: Q Qu, J Zhuang, H Ying; (II) Administrative support: H Ying; (III) Provision of study materials or patients: \\ JY Zhuang, L Yuan, YP Tang; (IV) Collection and assembly of data: J Zhuang, C Xu, L Yuan, Y Tang, Q Shu, Y Bao, H Han; (V) Data analysis and \\ interpretation: Q Qu, J Zhuang, Z Ai, H Ying; (VI) Manuscript writing: All authors; (VII) Final approval of manuscript: All authors. \\ "These authors contributed equally to this work. \\ Correspondence to: Hao Ying. Department of Obstetrics, Shanghai First Maternity and Infant Hospital, Tongji University School of Medicine, No. \\ 2699 Gaokexi Road, Shanghai 201204, China. Email: stephenying_2011@163.com.
}

Background: Our study aimed to reveal the relationship of maternal pentraxin 3 (PTX3)'s serum concentrations in early pregnancy with gestational diabetes mellitus (GDM) and to explore its potential in the prediction of GDM.

Methods: Totally 824 pregnant women were enrolled and divided into a GDM group and a normal glucose tolerance (NGT) group, whose maternal fasting serum PTX3 levels, plasma glucose and insulin were collected. The beta cell function index and quantitative insulin sensitivity check index (QUICKI) was calculated and a homeostatic model assessment of insulin resistance (HOMA-IR) was used with SPSS 22 software used for statistical analysis.

Results: Of all subjects, $13.59 \%$ developed GDM. Compared to the NGT group, the PTX3 level was increased in the GDM group (1.48 vs. $1.52 \mathrm{ng} / \mathrm{mL}, \mathrm{P}<0.05)$, and independently associated with the prediction of GDM (4.209, 95\% CI, 1.756-10.091) $(\mathrm{P}=0.001)$. The area under receiver operating characteristic curve (AUROC) of the combined screening of PTX3 for GDM was incremented to 0.657 by the addition of maternal characteristics, and it reached a maximum of 0.743 in further combination with biochemical markers.

Conclusions: Serum PTX3 levels in early pregnancy may provide a useful approach for early prediction of GDM.

Keywords: Gestational diabetes mellitus (GDM); pentraxin 3 (PTX3); prediction

Submitted Sep 15, 2019. Accepted for publication Nov 22, 2019.

doi: 10.21037/atm.2019.12.25

View this article at: http://dx.doi.org/10.21037/atm.2019.12.25

\section{Introduction}

Gestational diabetes mellitus (GDM) is a disease, in which develops carbohydrate intolerance during pregnancy (1). Women with GDM will increase the risk of preeclampsia and a cesarean delivery, their offspring also have an increased risk of birth trauma macrosomia, and shoulder dystocia $(2,3)$. Both mothers and their offspring have more likely developed cardiovascular disease, obesity, and type 2 diabetes mellitus (T2DM) (4-7).

Early intervention during pregnancy can significantly decrease the incidence of GDM, as well as less gestational weight gain and lower adverse outcomes of pregnancy, including hypertensive disorders, cesarean delivery and macrosomia (8-14). Sovio et al. indicated that before the diagnosis of GDM, there had already occurred accelerated fetal growth as early as 20 weeks of gestation (15). Furthermore, Logan et al. demonstrated that even mothers 


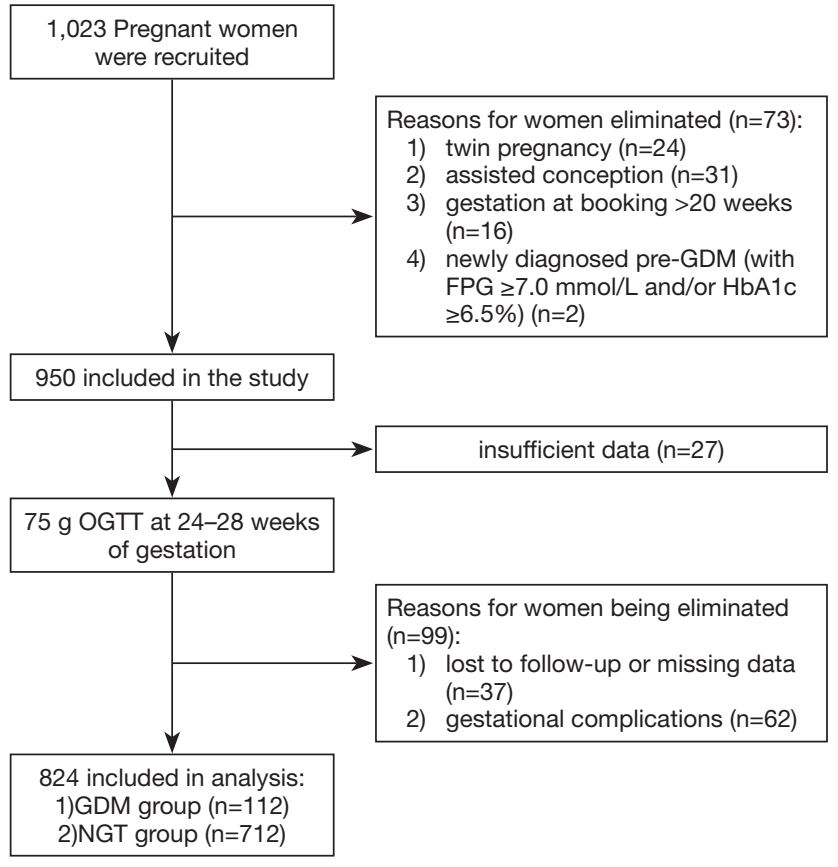

Figure 1 Flow diagram summarizing selection of patients.

with GDM had good glycemic control, their offspring also increased adiposity in early infancy (16). Early screening can improve glucose homeostasis and provide more time for intervention prior to term, so it is important to screen early in pregnancy to improve the outcome of GDM mothers and their fetus.

Recent evidence has suggested that the first trimester maternal serum levels of various biochemical factors are associated with GDM, including high-sensitivity C-reactive protein (hs-CRP), tumor necrosis factor- $\alpha$ (17), pregnancy-associated plasma protein A (18), placental growth factor (19), sex hormone-binding globulin, adiponectin and 1,5 anhydroglucitol (20), though not all were accurate as predictors.

In recent years, chronic subclinical inflammation is considered to have a potential role in the pathogenesis of hyperglycemia. Pentraxin 3 (PTX3), a recently identified multimeric inflammatory mediator, is correlated with the process of insulin resistance and its level is also increased in diabetes mellitus and diabetic nephropathy (21-23). Todoric et al. first observed that serum PTX3 concentrations are related to glucose levels in pregnant women with GDM and are negatively associated with insulin sensitivity, but their research focused on PTX3 serum concentrations at 24-28 weeks of gestation (24).
This study's first aim was to evaluate the relationship between the early maternal serum PTX3 and the occurrence of GDM at 24-28 weeks of gestation. The second goal was to measure the predictive value of PTX3 in early pregnancy in patients who developed GDM.

\section{Methods}

\section{Patient population and data collection}

A prospective cohort study was performed at Shanghai First Maternity and Infant Hospital, Tongji University School of Medicine, China between Oct 2015 and Dec 2015. We invited women with maternal age $\geq 18$ years, singleton primipara pregnancy, nonsmokers, with confirmed gestation $\leq 20$ weeks and spontaneous conception to participate our study. Women were excluded if they had multiple pregnancies, pre-GDM, hypertensive disorders, preterm delivery, cardiovascular disease, immunological disease, glucocorticoid therapy, or other severe illness. Women who were suffering from fetal abnormalities including chromosomally abnormal fetuses, structural defects or fetal growth restriction during pregnancy were also excluded.

On the first visit, held at $\leq 20$ weeks of gestation, we recorded maternal characteristics and medical history and gestational age was confirmed. The levels of fasting serum PTX3 concentrations, fasting plasma glucose (FPG), glycosylated hemoglobin (HbA1c) and fasting plasma insulin (FINS) were tested at the first routine checkup. Additionally, during 24-28 weeks, all subjects accepted a $75 \mathrm{~g}$ OGTT. The study was approved by the Ethics Committee of our hospital (KS1535), and all participants signed the informed consent. The clinical trial registration number is NCT03563638.

A flow diagram describing the selection of women is shown in Figure 1. In total, 1,023 women with gestation $\leq 20$ weeks were recruited. Excluded from the study were 199 women owing to twin pregnancy $(\mathrm{n}=24)$, assisted conception $(\mathrm{n}=31)$, gestation at booking $>20$ weeks $(\mathrm{n}=16)$, insufficient data $(\mathrm{n}=27)$, loss to follow-up or having missing data $(\mathrm{n}=37)$, gestational complications $(n=62)$ or pre-GDM $(n=2)$. The remaining 824 women were participated in the study.

\section{GDM diagnostic criteria}

GDM was verified in a one-step approach based on the International Association of Diabetes and Pregnancy Study Groups (IADPSG) criteria (25). GDM is diagnosed when 
any of the plasma glucose values are met or exceeded: FPG $\geq 5.1 \mathrm{mmol} / \mathrm{L}, 1 \mathrm{~h}-\mathrm{PG} \geq 10.0 \mathrm{mmol} / \mathrm{L}$ or $2 \mathrm{~h}-\mathrm{PG} \geq 8.5 \mathrm{mmol} / \mathrm{L}$.

\section{Laboratory measurements}

We collected a venous blood sample from each participant at the first routine checkup. We separated the serum samples, and then stored them at $-80{ }^{\circ} \mathrm{C}$ until we analyzed the levels of serum PTX3. At the same time, FPG, HbA1c and FINS were also tested.

Venous blood specimen collection of each woman was gathered at the first routine checkup, and the blood serum were then separated and stored at $-80^{\circ} \mathrm{C}$. The levels of PTX3, FPG, HbA1c and FINS from the samples were tested and analyzed subsequently. FPG was tested by G-6-PDH method, HbAlc was tested by HPLC method, and FINS was tested by Access Ultrasensitive Insulin method. The levels of serum PTX3 was tested by the same method of Akhter et al. (26). Serum PTX3 was analyzed using commercial sandwich ELISA kits (DY1826, R\&D Systems, Minneapolis, MN, USA). The detection limit was $0.16 \mathrm{ng} / \mathrm{mL}$. The intra-assay coefficient of variation (CV) was $10 \%$ and the inter-assay CV was $15 \%$ for the PTX3 assay.

\section{Anthropometric measurements and data calculations}

Pre-pregnant weight (in $\mathrm{kg}$ ) was divided by height (in $\mathrm{m}$ ) squared to count body mass index (BMI). After resting for five minutes in the sitting position, systolic blood pressure (SBP) and diastolic blood pressure (DBP) of each woman were measured on the right arm with an automated sphygmomanometer. $[\mathrm{SBP}(\mathrm{mmHg})+2 \times \mathrm{DBP}$ $(\mathrm{mmHg})] / 3$ was used to calculate mean arterial pressure (MAP). A HOMA2 calculator, released by the Diabetes Trials Unit affiliated to the University of Oxford, were employed to calculate homeostatic model assessment of insulin resistance (HOMA-IR) and HOMA- $\beta$ data, which is available at: http://www.dtu.ox.ac.uk/homacalculator/ index.php (updated January 8, 2013). Quantitative insulin sensitivity check index (QUICKI) used by the last equation, was calculated as $1 /[\log (\mathrm{FINS})+\log (\mathrm{FPG})]$, as previously described by Katz et al. (27). The trapezoidal rule was applied to determine the AUCglu.

\section{Statistical analyses}

Continuous variables were summarized with mean \pm standard deviation (mean $\pm \mathrm{SD}$ ) or median (inter-quartile range, IQR), while between-group comparisons were conducted by $t$ tests or Wilcoxon rank tests according to assessments of normality and homogeneity of variance. Categorical variables were summarized with percentages, and two groups compared by the use of a chi-square test or Fisher's exact test as appropriate. Spearman correlation analyses were carried out to analyze relationships between PTX3 and other variables. Logistic regression analysis was fitted to analyze the contributions of variables including PTX 3 and adjusted for potential factors. Specificity, sensitivity, positive and negative likelihood ratios, as well as positive and negative predictive values of PTX3 concentrations were calculated using tentative PTX3 thresholds to estimate the ability of PTX3 alone to serve as a predictor of GDM. Cut-off values of PTX3, HbA1c and FIN were determined by receiver operating characteristics (ROC) analysis using the Youden index. The predictive ability of each model was inferred using the area under the curve (AUROC). SPSS Statistics version 22.0 software was used to analyze all data. The difference was statistically significant when a $\mathrm{P}$ value $<0.05$. An AUROC $>0.70$ was defined as a significant discrimination.

\section{Results}

\section{Clinical characteristics of the population}

Of the 824 participants completing the study, 112 (13.59\%) were diagnosed with GDM during 24-28 weeks of gestation. The median gestational weeks at the time of blood drawing for serum PTX3 test was 14.86 weeks (ranging from 10 to 20 weeks). Women who subsequently developed GDM, compared to those unaffected, were older ( $\mathrm{P}=0.001)$, had higher HOMA- $\beta(\mathrm{P}=0.02)$, HbA1c $(\mathrm{P}=0.001)$, MAP $(\mathrm{P}=0.030)$, FINS and HOMA-IR levels $(\mathrm{P}=0.004)$, a lower QUICKI level $(\mathrm{P}<0.001)$, more often a firstdegree relative history of diabetes $(\mathrm{P}=0.001)$, while the prepregnancy BMI ,FBG and the gestational weight gain up to the OGTT were similar between the outcome groups. Additionally, after adjustment for maternal age, MAP and family history of diabetes in a first-degree relative, the fasting PTX3 values in early pregnancy were statistically significantly higher in GDM subjects $(\mathrm{P}=0.011)$ (Table 1).

\section{Relationship between PTX 3 and relevant parameters in the 824 study participants}

Spearman correlation analyses showed that PTX3 was 
Table 1 Comparison of clinical characteristics between the two groups $(\mathrm{n}=824)$

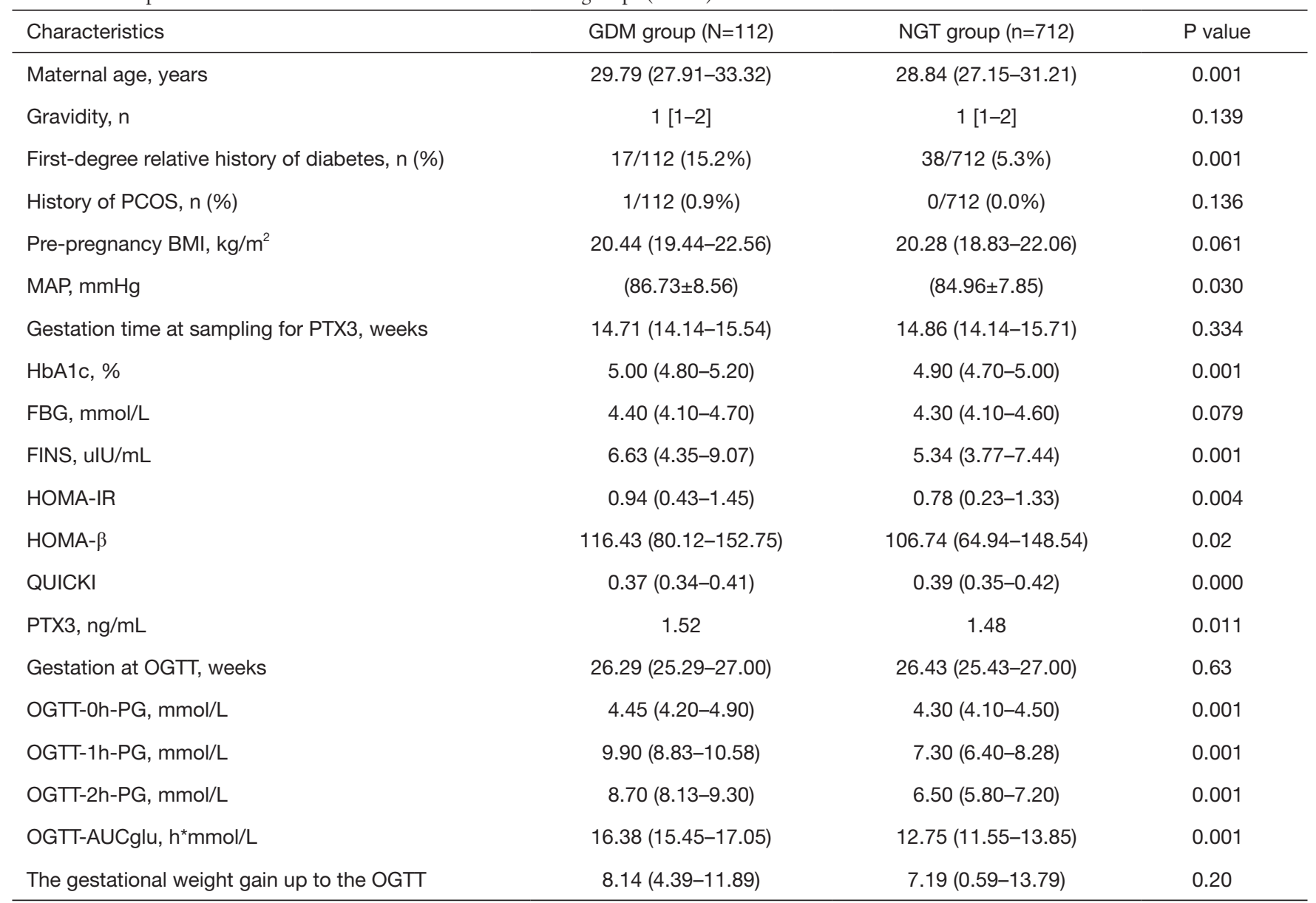

Data are expressed as median (IQR), number (\%), or mean \pm SE. Comparisons between outcome groups: Wilcoxon rank test or $t$-test for continuous variables, $\chi^{2}$ test and Fisher's exact test for categorical variables. $0 \mathrm{~h}-\mathrm{PG}$, fasting plasma glucose before glucose challenge; $1 \mathrm{~h}$ PG, 1 hour postprandial plasma glucose; 2h-PG, 2-hour postprandial plasma glucose; AUCglu, area under the curve of glucose during OGTT; BMI, body mass index; FBG, fasting blood glucose; FINS, fasting insulin; HbA1c, glycated hemoglobin; HOMA-IR, homeostasis model assessment-insulin resistance index; HOMA- $\beta$, homeostasis model assessment beta cell function index; MAP, mean maternal pressure; OGTT, oral glucose tolerance test; PCOS, polycystic ovary syndrome; QUICKI, quantitative insulin sensitivity check index; GDM, gestational diabetes mellitus.

positively associated with FINS ( $\mathrm{r}=0.132, \mathrm{P}=0.001)$, 0hPG ( $r=0.075, P=0.032)$, 1h-PG ( $r=0.086, P=0.014)$, and AUCglu ( $\mathrm{r}=0.092, \mathrm{P}=0.009)$ during OGTT, but negatively correlated with QUICKI $(\mathrm{r}=-0.133, \mathrm{P}=0.001)$. After adjusting for maternal age, MAP and family history of diabetes, there was no statistically significant relationship between PTX3 levels with HbA1c, FPG or 2h-PG at the time of the OGTT (Table 2).

\section{Evaluation of PTX3 for GDM}

Hierarchical analysis was conducted for further assessment of PTX3 by percentile (Table 3). When the PTX3 level was below the 5 th percentile $(1.05 \mathrm{ng} / \mathrm{mL})$ or the 10 th percentile $(1.12 \mathrm{ng} / \mathrm{mL})$, the incidence of GDM was $0.00 \%$ and $1.20 \%$ respectively, the negative predictive rate was $100.0 \%$ and $98.8 \%$, the false negative rate was $0.0 \%$ and $0.9 \%$, and the negative likelihood ratio was 0.00 and 0.08 , or $<0.10$ for both. When the PTX3 level was below the 15 th percentile $(1.18 \mathrm{ng} / \mathrm{mL})$, a parallel result was obtained. At this cut-off point for PTX3 (PTX $3_{\text {cutoff }}$ ) of $1.21 \mathrm{ng} / \mathrm{mL}$, the sensitivity of the prediction for GDM was $94.6 \%$, the specificity was $20.2 \%$, the negative predictive rate was $96.0 \%$, and the negative likelihood ratio was 0.26 (Table 4). 
Table 2 Association between PTX3 and clinical parameters in 824 study participants

\begin{tabular}{lcc}
\hline Parameter & Spearman correlation coefficient & P value \\
\hline HbA1c & -0.021 & 0.548 \\
FBG & 0.041 & 0.245 \\
FINS & 0.132 & 0.001 \\
HOMA-IR & 0.067 & 0.071 \\
HOMA- $\beta$ & 0.054 & 0.143 \\
QUICKI & -0.133 & 0.001 \\
Oh-PG at OGTT & 0.075 & 0.032 \\
1h-PG at OGTT & 0.086 & 0.014 \\
2h-PG at OGTT & 0.067 & 0.055 \\
AUCglu at OGTT & 0.092 & 0.009 \\
\hline
\end{tabular}

Oh-PG, fasting plasma glucose before glucose challenge; 1h-PG, 1 hour postprandial plasma glucose; 2h-PG, 2-hour postprandial plasma glucose; AUCglu, area under the curve of glucose during OGTT; FBG, fasting blood glucose; FINS, fasting insulin; HbA1c, glycated hemoglobin; HOMA-IR, homeostasis model assessment-insulin resistance index; HOMA- $\beta$, homeostasis model assessment beta cell function index; OGTT, oral glucose tolerance test; QUICKI, quantitative insulin sensitivity check index.

Compared to women with serum PTX3 $<1.21 \mathrm{ng} / \mathrm{mL}$ (PTX3 $3_{\text {cutoff }}$ ), women with PTX3 $\geq 1.21 \mathrm{ng} / \mathrm{mL}$ in early pregnancy were 4.209 (95\% CI, 1.756-10.091) times more likely to develop GDM (Table 4).

ROC analyses were performed to evaluate the usefulness of serum PTX3 levels for the prediction of GDM. The AUROC for prediction of GDM based on maternal characteristics incremented from 0.602 to 0.657 after the inclusion of PTX3, and reached a maximum of 0.743 after additional combination with biochemical markers including HbA1c and FINS (Figure 2).

\section{Multivariate logistic regression analysis}

We investigated the prediction GDM with the observed parameters using a multiple regression model (Table 4). According to the odds ratios, five variables were significantly and independently related to GDM including maternal age [adjusted odds ratio (AOR) for $\geq 35$ years old, 4.235 (95\% CI, 2.051-8.744); $\mathrm{P}<0.001$ ], PTX3 [AOR for cutoff value, 4.209 (95\% CI, 1.756-10.091); $\mathrm{P}=0.001$ ], firstdegree relative history of diabetes [AOR 3.604 (95\% CI,
1.547-6.068); $\mathrm{P}=0.001$ ], HbA1c [AOR for cutoff value, 2.833 (95\% CI, 1.798-4.464); $\mathrm{P}<0.001]$, FINS [AOR for cutoff value, 1.919 (95\% CI, 1.209-3.047); $\mathrm{P}=0.006$ ]. There were no significant contributions from a history of polycystic ovary syndrome, pre-pregnancy BMI $\geq 24 \mathrm{~kg} / \mathrm{m}^{2}$ or FPG (cutoff value $4.65 \mathrm{mmol} / \mathrm{L}$ ). All the cutoff values were based on the largest Youden index.

\section{Discussion}

The principal findings of this study were: (I) maternal serum levels of PTX3 in early pregnancy are significantly increased in pregnant women who develop GDM; (II) the AUROC for prediction of GDM based on PTX3 and other factors was 0.73 ; (III) PTX3 $<1.21 \mathrm{pg} / \mathrm{mL}$ may be useful to rule out the diagnosis of GDM.

The prevalence of GDM in our study is $13.59 \%$, and it was similar to other studies $(28,29)$. PTX3 is produced in macrophages, granulocytes and endothelial cells, at inflammation's area. PTX3 together with the hs-CRP and other acute phase proteins, is an essential component of the humoral arm of innate immunity, and belong to the pentraxins' superfamily (30). PTX3 reflects tissue inflammation and damage under diverse clinical conditions (31). Syngelaki et al. showed that the levels of serum hs-CRP are not useful for first-trimester screening for GDM $(17,20)$. A growing number of studies on the relationship between PTX3 and hyperglycemia associated with insulin resistance have been published that suggest potential involvement of PTX3 in diabetes mellitis pathology $(21-24,32)$. PTX3 is a good predictor because its level in early pregnancy increases slightly but the change is limited (33). Furthermore, because PTX3 is related with the inflammation of vascular, PTX3 has been considered to associate with preeclampsia (34) and type 1 diabetes (35), and PTX3 levels are related to future cardiovascular disease risk (36). Therefore, we hypothesized that in the first trimester, the level of PTX3 as an inflammatory marker involved in pathogenesis of diabetes related comorbidities could be correlated with the development of GDM during pregnancy.

Our study shows that PTX3 level during pregnancy was significantly higher in GDM group. There was a significant positive correlation between serum glucose and PTX3, and a negative link with the QUICKI. These outcomes indicated that PTX3 may be associated with insulin resistance, which may have a role in the mechanism of development of GDM. PTX3 was negatively correlated 
Table 3 Evaluation index of different PTX3 percentiles for GDM

\begin{tabular}{|c|c|c|c|c|c|c|c|c|c|c|c|}
\hline PTX3 (ng/mL) & \multicolumn{2}{|c|}{ Incidence of GDM, \% } & Sensitivity, \% & Specificity, \% & Youden's index & FPR, \% & FNR, \% & PPV, \% & NPV, \% & $+\mathrm{LR}$ & $-\mathrm{LR}$ \\
\hline P5 $=1.05$ & 0 & 14.3 & 100 & 5.6 & 0.06 & 94.4 & 0 & 14.3 & 100 & 1.06 & 0 \\
\hline $\mathrm{P} 10=1.12$ & 1.2 & 15 & 99.1 & 11.4 & 0.1 & 88.6 & 0.9 & 15 & 98.8 & 1.12 & 0.08 \\
\hline $\mathrm{P} 15=1.18$ & 4.1 & 15.3 & 95.5 & 16.6 & 0.12 & 83.4 & 4.5 & 15.3 & 95.9 & 1.15 & 0.27 \\
\hline P20 =1.23 & 6.1 & 15.5 & 91.1 & 21.8 & 0.13 & 78.2 & 8.9 & 15.5 & 93.9 & 1.16 & 0.41 \\
\hline $\mathrm{P} 25=1.27$ & 8.3 & 15.4 & 84.8 & 26.5 & 0.11 & 73.5 & 15.2 & 15.4 & 91.7 & 1.16 & 0.57 \\
\hline P30 =1.32 & 9.7 & 15.3 & 78.6 & 31.3 & 0.1 & 68.7 & 21.4 & 15.3 & 90.3 & 1.14 & 0.68 \\
\hline P35 =1.35 & 10.4 & 15.3 & 73.2 & 36.2 & 0.09 & 63.8 & 26.8 & 13.6 & 89.6 & 1.15 & 0.73 \\
\hline $\mathrm{P} 50=1.49$ & 11.7 & 15.5 & 57.1 & 51.1 & 0.08 & 48.9 & 42.9 & 15.5 & 88.3 & 1.17 & 0.84 \\
\hline P55 =1.53 & 12.6 & 14.8 & 49.1 & 55.6 & 0.05 & 44.4 & 50.9 & 14.8 & 87.4 & 1.11 & 0.92 \\
\hline P75 =1.79 & 12.8 & 16.0 & 29.5 & 75.7 & 0.05 & 24.3 & 70.5 & 16.0 & 87.2 & 1.21 & 0.93 \\
\hline P85 =1.95 & 12.7 & 18.7 & 20.5 & 86.0 & 0.06 & 14.0 & 79.5 & 18.7 & 87.3 & 1.46 & 0.92 \\
\hline P95 $=1.44$ & 13.3 & 19.5 & 7.1 & 95.4 & 0.03 & 4.6 & 92.9 & 19.5 & 86.7 & 1.54 & 0.97 \\
\hline
\end{tabular}

+LR, positive likelihood ratio; -LR, negative likelihood ratio; FNR, false negative rate, FPR, false positive rate, NPV, negative predictive rate, PPV, positive predictive rate; GDM, gestational diabetes mellitus.

Table 4 Multivariate logistic regression analysis for the prediction of GDM by all parameters

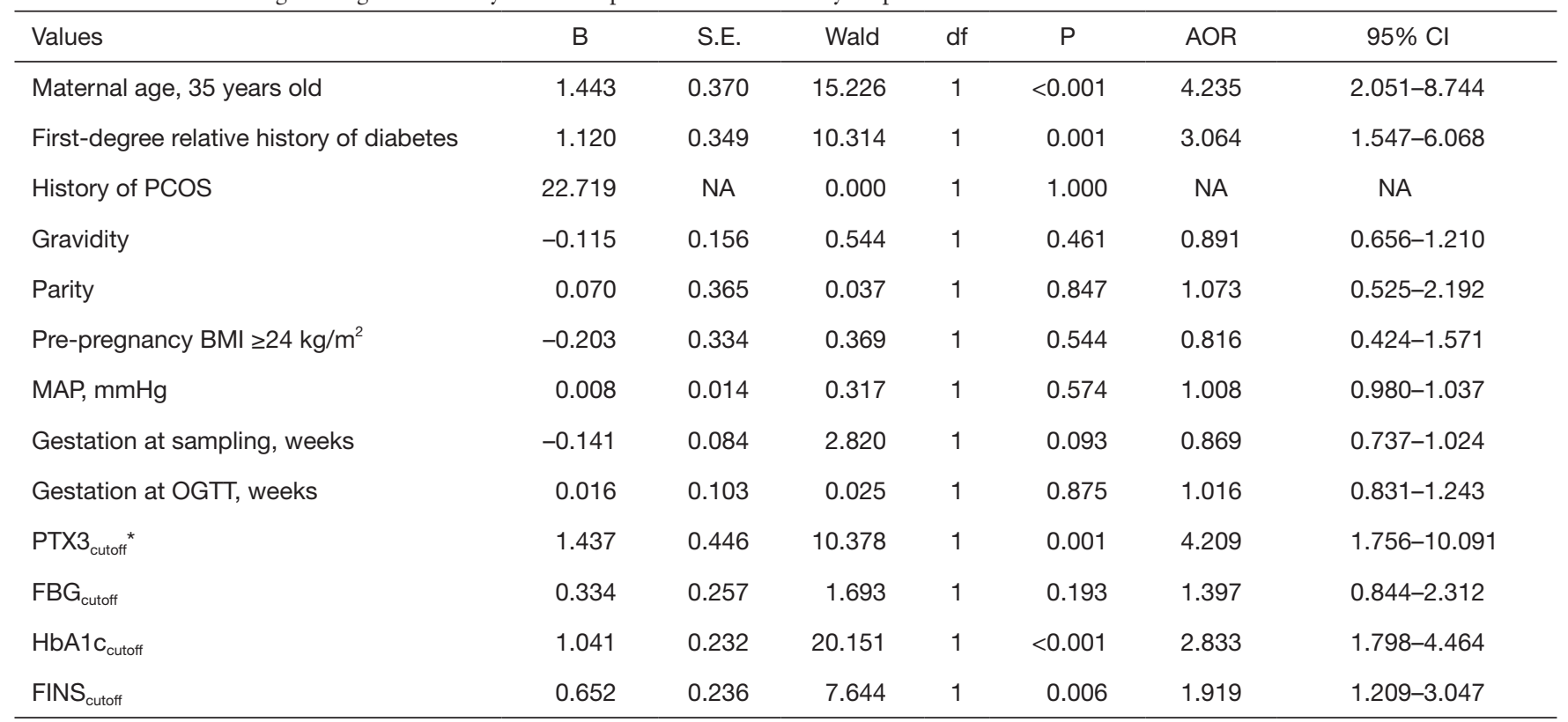

Overweight was defined as $\mathrm{BMI} \geq 24.0 \mathrm{~kg} / \mathrm{m}^{2} ;{ }^{*}, \mathrm{PTX}{ }_{\text {cutoff }}=1.21 \mathrm{ng} / \mathrm{mL}$. BMI, body mass index; HbA1c, glycated hemoglobin; FPG, fasting plasma glucose; FINS, fasting insulin; MAP, mean maternal pressure; NA, not available; OGTT, oral glucose tolerance test; PCOS, polycystic ovary syndrome. GDM, gestational diabetes mellitus. 


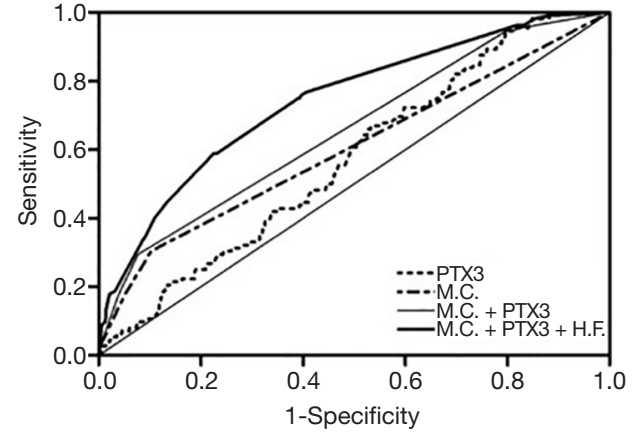

Figure 2 ROC curves of different combination screening for GDM. H.F., HbA1c and FINS; M.C., maternal characteristics (maternal age $\geq 35$ years old, first-degree relative history of diabetes). PTX3, pentraxin 3; GDM, gestational diabetes mellitus.

with QUICKI, which showed that a higher level of PTX3 is related to lower insulin sensitivity, but more studies are needed to determine the mechanism. The present finding is in line with other recent studies $(24,37)$.

Publications in the literature have reported that potential predictors of GDM, when used alone for screening, have some shortcomings, such as a low detection rate and high false positive rate. To make up for the limitations of single screenings, a variety of blood indicators have been used in combined screening for $\operatorname{GDM}(38,39)$. Our multiple logistic regression analysis on risk factor assessment of GDM revealed that five variables remained statistically significant among all the parameters (age of women, family history of diabetes in first-degree relatives, PTX3, HbA1c and FINS, $\mathrm{P}<0.05)$. Further, the AUROC for prediction of GDM based on maternal characteristics was improved after combination with PTX3, and reached a maximum of 0.743 with combination of other biochemical markers and characteristics of the mother. Such an elevation of AUROC after combining it with several clinical parameters is also consistent with reports in the literature $(38,39)$.

One study reported different results from others. Lekva et al. (36) reported that PTX3 levels was significantly lower in women with GDM than those without GDM, not only at pregnancy but also at a 5 -year follow-up by using the WHO criteria. This discrepancy could be due to fewer patients with GDM, the different GDM diagnostic criteria (WHO and IADPSG), and the screening time of OGTT at 30-32 weeks. The study also included preeclampsia, while our study excluded this disease.

An important strength of our study is that it was the first to examine PTX3 for the early prediction of GDM, and detected a significant increase of fasting serum PTX3 concentration in pregnant women who develop GDM, which may contribute to the early prediction of GDM. In addition, unlike other studies, we have identified a predictor; serum PTX3 $<1.21 \mathrm{pg} / \mathrm{mL}$ may be useful to rule out the diagnosis of GDM. Therefore, if this finding is corroborated in future studies with more participants, women with lower levels will not need any further procedures to rule out GDM.

There are some limitations of this study. Its major shortcoming is that we have not examined the level of hsCRP at the same time in different participants. Hs-CRP is also a classical inflammatory marker, and some research in the first trimester has suggested that hs-CRP might be a marker for prediction of GDM (40). Second, our study was not a sequential cohort study; we only collected values for different women at their first visit in different weeks of gestation, not throughout their entire pregnancy or after long-term follow-up. Third, further studies are required to investigate whether our results are reproducible at larger sample sizes.

\section{Conclusions}

We first demonstrated that serum PTX3 levels are an early predictor of GDM, and unlike other studies, lower serum PTX3 levels $(<1.21 \mathrm{pg} / \mathrm{mL})$ may be useful to rule out a diagnosis of GDM.

\section{Acknowledgments}

We gratefully acknowledge the participants and Associate Professor Zisheng Ai from Tongji University who gave advice and suggestions on various aspects of the statistical analysis. The study was supported by the Maternal Medicine of Shanghai First Maternity and Infant Hospital, Tongii University, Shanghai, China.

Funding: This study was funded by a research grant from the Key Project of National Natural Science Foundation (grant numbers 81771600), National Health and Family Planning Commission of the People's Republic of China (grant numbers 201540256), Shanghai Municipal Commission of Health and Family Planning (grant numbers 201740109), Shanghai Hospital Development Center (grant numbers SHDC12017120), Shanghai Science and Technology Committee (grant numbers 17411950600) and Shanghai Training and Support Program for Outstanding Young 
Medical Talents.

\section{Footnote}

Conflicts of Interest: The authors have no conflicts of interest to declare.

Etbical Statement: The authors are accountable for all aspects of the work in ensuring that questions related to the accuracy or integrity of any part of the work are appropriately investigated and resolved. The study was approved by the Ethics Committee of our hospital (KS1535), and all participants signed the informed consent.

\section{References}

1. Egan AM, Dunne FP. Optimal management of gestational diabetes. Br Med Bull. 2019;131:97-108.

2. Committee on Practice Bulletins-Obstetrics. Practice Bulletin No. 180: Gestational Diabetes Mellitus. Obstet Gynecol 2017;130:e17-37.

3. Hocaoglu M, Demirer S, Senturk H, et al. Differential expression of candidate circulating microRNAs in maternal blood leukocytes of the patients with preeclampsia and gestational diabetes mellitus. Pregnancy Hypertens 2019;17:5-11.

4. Metzger BE. Long-term outcomes in mothers diagnosed with gestational diabetes mellitus and their offspring. Clin Obstet Gynecol 2007;50:972-9.

5. Boney CM, Verma A, Tucker R, et al. Metabolic syndrome in childhood: association with birth weight, maternal obesity, and gestational diabetes mellitus. Pediatrics 2005;115:e290-6.

6. Harreiter J, Dovjak G, Kautzky-Willer A. Gestational diabetes mellitus and cardiovascular risk after pregnancy. Womens Health (Lond) 2014;10:91-108.

7. Smith GN, Louis JM, Saade GR. Pregnancy and the Postpartum Period as an Opportunity for Cardiovascular Risk Identification and Management. Obstet Gynecol 2019;134:851-62.

8. Bartha JL, Martinez-Del-Fresno P, Comino-Delgado R. Early diagnosis of gestational diabetes mellitus and prevention of diabetes-related complications. Eur J Obstet Gynecol Reprod Biol 2003;109:41-4.

9. Alunni ML, Roeder HA, Moore TR, et al. First trimester gestational diabetes screening - Change in incidence and pharmacotherapy need. Diabetes Res Clin Pract 2015;109:135-40.
10. Seshiah V, Cynthia A, Balaji V, et al. Detection and care of women with gestational diabetes mellitus from early weeks of pregnancy results in birth weight of newborn babies appropriate for gestational age. Diabetes Res Clin Pract 2008;80:199-202.

11. Horie I, Kawasaki E, Sakanaka A, et al. Efficacy of nutrition therapy for glucose intolerance in Japanese women diagnosed with gestational diabetes based on IADPSG criteria during early gestation. Diabetes Res Clin Pract 2015;107:400-6.

12. Palatnik A, Mele L, Landon MB, et al. Timing of treatment initiation for mild gestational diabetes mellitus and perinatal outcomes. Am J Obstet Gynecol 2015;213:560.e1-8.

13. Thangaratinam S, Rogozinska E, Jolly K, et al. Effects of interventions in pregnancy on maternal weight and obstetric outcomes: meta-analysis of randomised evidence. BMJ 2012;344:e2088.

14. Koivusalo SB, Rono K, Klemetti MM, et al. Gestational Diabetes Mellitus Can Be Prevented by Lifestyle Intervention: The Finnish Gestational Diabetes Prevention Study (RADIEL): A Randomized Controlled Trial. Diabetes Care 2016;39:24-30.

15. Sovio U, Murphy HR, Smith GC. Accelerated Fetal Growth Prior to Diagnosis of Gestational Diabetes Mellitus: A Prospective Cohort Study of Nulliparous Women. Diabetes Care 2016;39:982-7.

16. Logan KM, Emsley RJ, Jeffries S, et al. Development of Early Adiposity in Infants of Mothers With Gestational Diabetes Mellitus. Diabetes Care 2016;39:1045-51.

17. Syngelaki A, Visser GH, Krithinakis K, et al. First trimester screening for gestational diabetes mellitus by maternal factors and markers of inflammation. Metabolism 2016;65:131-7.

18. Savvidou MD, Syngelaki A, Muhaisen M, et al. First trimester maternal serum free beta-human chorionic gonadotropin and pregnancy-associated plasma protein A in pregnancies complicated by diabetes mellitus. BJOG 2012;119:410-6.

19. Syngelaki A, Kotecha R, Pastides A, et al. First-trimester biochemical markers of placentation in screening for gestational diabetes mellitus. Metabolism 2015;64:1485-9.

20. Corcoran SM, Achamallah N, Loughlin JO, et al. First trimester serum biomarkers to predict gestational diabetes in a high-risk cohort: Striving for clinically useful thresholds. Eur J Obstet Gynecol Reprod Biol 2018;222:7-12.

21. Yilmaz MI, Axelsson J, Sonmez A, et al. Effect of renin 
angiotensin system blockade on pentraxin 3 levels in type-2 diabetic patients with proteinuria. Clin J Am Soc Nephrol 2009;4:535-41.

22. Suliman ME, Yilmaz MI, Carrero JJ, et al. Novel links between the long pentraxin 3, endothelial dysfunction, and albuminuria in early and advanced chronic kidney disease. Clin J Am Soc Nephrol 2008;3:976-85.

23. Inforzato A, Reading PC, Barbati E, et al. The "sweet" side of a long pentraxin: how glycosylation affects PTX3 functions in innate immunity and inflammation. Front Immunol 2013;3:407.

24. Todoric J, Handisurya A, Knapp B, et al. Relationship of pentraxin 3 with insulin sensitivity in gestational diabetes. Eur J Clin Invest 2013;43:341-9.

25. International Association of Diabetes and Pregnancy Study Groups Consensus Panel, Metzger BE, Gabbe SG, et al. International association of diabetes and pregnancy study groups recommendations on the diagnosis and classification of hyperglycemia in pregnancy. Diabetes Care 2010;33:676-82.

26. Akhter T, Wikstrom AK, Larsson M, et al. Serum Pentraxin 3 is associated with signs of arterial alteration in women with preeclampsia. Int J Cardiol 2017;241:417-22.

27. Katz A, Nambi SS, Mather K, et al. Quantitative insulin sensitivity check index: a simple, accurate method for assessing insulin sensitivity in humans. J Clin Endocrinol Metab 2000;85:2402-10.

28. Lee KW, Ching SM, Ramachandran V, et al. Prevalence and risk factors of gestational diabetes mellitus in Asia: a systematic review and meta-analysis. BMC Pregnancy Childbirth 2018;18:494.

29. Guariguata L, Linnenkamp U, Beagley J, et al. Global estimates of the prevalence of hyperglycaemia in pregnancy. Diabetes Res Clin Pract 2014;103:176-85.

30. Bonacina F, Baragetti A, Catapano AL, et al. Long pentraxin 3: experimental and clinical relevance in cardiovascular diseases. Mediators Inflamm

Cite this article as: $\mathrm{Qu} \mathrm{X}$, Zhuang $\mathrm{J}, \mathrm{Xu} \mathrm{C}$, Ai Z, Yuan L, Tang Y, Shu Q, Bao Y, Han H, Ying H. Maternal serum pentraxin 3 level in early pregnancy for prediction of gestational diabetes mellitus. Ann Transl Med 2019;7(23):722. doi: 10.21037/ atm.2019.12.25
2013;2013:725102.

31. Mantovani A, Garlanda C, Doni A, et al. Pentraxins in innate immunity: from C-reactive protein to the long pentraxin PTX3. J Clin Immunol 2008;28:1-13.

32. Miyaki A, Choi Y, Maeda S. Pentraxin 3 production in the adipose tissue and the skeletal muscle in diabetic-obese mice. Am J Med Sci 2014;347:228-33.

33. Larsson A, Palm M, Helmersson J, et al. Pentraxin 3 values during normal pregnancy. Inflammation 2011;34:448-51.

34. Zhou P, Luo X, Qi HB, et al. The expression of pentraxin 3 and tumor necrosis factor-alpha is increased in preeclamptic placental tissue and maternal serum. Inflamm Res 2012;61:1005-12.

35. Castiglioni MT, Scavini M, Cavallin R, et al. Elevation of plasma levels of the long pentraxin 3 precedes preeclampsia in pregnant patients with type 1 diabetes. Autoimmunity 2009;42:296-8.

36. Lekva T, Michelsen AE, Bollerslev J, et al. Low circulating pentraxin 3 levels in pregnancy is associated with gestational diabetes and increased apoB/apoA ratio: a 5-year follow-up study. Cardiovasc Diabetol 2016;15:23.

37. Yildirim M, Simavli SA, Uysal Derbent A, et al. Is There Any Relationship between Plasma Pentraxin 3 Levels and Gestational Diabetes Mellitus? Gynecol Obstet Invest 2015;80:223-7.

38. Savvidou M, Nelson SM, Makgoba M, et al. First-trimester prediction of gestational diabetes mellitus: examining the potential of combining maternal characteristics and laboratory measures. Diabetes 2010;59:3017-22.

39. Maitland RA, Seed PT, Briley AL, et al. Prediction of gestational diabetes in obese pregnant women from the UK Pregnancies Better Eating and Activity (UPBEAT) pilot trial. Diabet Med 2014;31:963-70.

40. Oztas E, Ozler S, Ersoy E, et al. Prediction of gestational diabetes mellitus by first trimester serum secreted frizzlerelated protein-5 levels. J Matern Fetal Neonatal Med 2016;29:1515-9. 